\title{
QUERY BY IMAGE MEDICAL TRAINING Optical Biopsy with Confocal Endoscopy (OB-CEM)
}

\author{
Olga Ferrer ${ }^{1}$, Vinicius Duval ${ }^{2}$, Jaime Delgado ${ }^{3}$, Claudio Rolim ${ }^{2}$ and Ruben Tous ${ }^{3}$ \\ ${ }^{1}$ University of La Laguna, UNESCO Chair of Telemedicine, Full Professor of Pathology \\ La Cuesta, La Laguna 38071, Canary Islands, Spain \\ \{catai\}@teide.net \\ http://www.teide.net/catai; http://www.catai.net/blog \\ ${ }^{2}$ University do Rio Grande do Sul, Brazil \\ \{vinids\}@terra.br \\ ${ }^{3}$ Universitat Politecnica de Catalunya (UPC-BARCELONATECH), Spain \\ \{jaime.delgado;rtous\}@ac.upc.edu
}

Keywords: $\quad$ Optical Biopsy. Query by image. ISO-15938-12, MPEG Query Format, MPQF, ISO 24800-3, JPSearch, JPEG Query Format, JPQF, Artificial Intelligence. Multimedia standard.

\begin{abstract}
The use of Optical Biopsies-OB (in the present case Confocal endomicroscopy-CEM) is limited due to difficulties to interpret images. The OB-CEM are taken by endoscopists, not trained in microscopic morphology which is the domain of the surgical pathology. To gain diagnostic confidence the endoscopists could consult the images to a pathologist or could use the technique proposed in the paper. That is, to search for similar images on Internet to compare the diagnosis.

The present paper is a positioning paper of how to build a CEM-image metadata to be used by the multimedia standards ISO-15938-12:2008 and ISO-24800-3 in order to search on line using a "query by image". Metadata semantics based on Kudo colorectal crypt architecture was used for annotation or automatic image extraction. The training set was composed of 25 OB-CEM chromo-colonoscopy images taken with a FICE (Fujinon Intelligent Chromoendoscopy). Those parameters were, whenever possible, automatically extracted from the image and included in the metadata for image mining. Future developments will annotate histological images is such a way that the query could also retrieve the histological image.
\end{abstract}

\section{INTRODUCTION}

An optical biopsy (OB)(Wang and VanDam, 2004) is a non-intrusive optic diagnostic method, capable to analyze the tissue in surface and in deepness with one of the following techniques: laser, OCT, infrared, fluorescence, spectroscopy etc. This means, that it is not necessary to extract the tissue from the body. Tissue is accessed through the surface of the body through the skin or by endoscopy.

In OBs the images are obtained in real time together with complementary information that allows evaluating the disease in vivo, but "goldenstandards" are still lacking (Ferrer-Roca, 2008) in contrast with those of the pathologist based on the histology of the normal fixed tissue (death tissue). OB-CEM is a confocal microscopy that obtains histological images closer to the field and training of pathologists than endoscopists in charge of the tech- nique (Ferrer-Roca, 2009) . It is therefore reasonable the lack of confidence on their interpretation. To solve the problem two methods could be defined: (1) a teleconsultation with a pathologist or (2) a nonsupervised search for a "similar image" on the Net using multimedia query and image mining techniques (R. Tous, 2008).

Standardization efforts to annotate, search and retrieve digital images are now a day taking place. Two of the more relevant initiatives are the MPEG Query Format (MPQF) (R. Tous , 2008) (ISO/IEC 15938-12:2008, 2008) and the JPEG's JPSearch project (R. Tous, 2008), (ISO/IEC 24800-3:2008, 2008). While MPQF has already reached its last standardization level, the JPSearch (whose Part 3, named JPSearch Query Format or simply JPQF, is just a profile of MPQF) is still an ongoing work, and faces the difficult challenge to provide an interoperable architecture for images' metadata management. 
Table 1: Modified KUDO criteria. Taken from Kiesslich (Kiesslich et al., 2008).

\begin{tabular}{|c|c|c|c|}
\hline Pit type & Characteristics & Apperance & Pit size \\
\hline $\mathbf{I}$ & Normal round & 000 & $\begin{array}{cc}0.07 & \pm \\
0.02 \mathrm{~mm} & \end{array}$ \\
\hline II & Stellate or papillary & & $\begin{array}{c}0.09 \quad \pm \\
0.02 \mathrm{~mm}\end{array}$ \\
\hline IIIs & $\begin{array}{l}\text { Tubular/round pits smaller then } \\
\text { type I }\end{array}$ & & $\begin{array}{c}0.03 \quad \pm \\
0.01 \mathrm{~mm}\end{array}$ \\
\hline III & Tubular large & & $\begin{array}{c}0.22 \\
0.09 \mathrm{~mm}\end{array}$ \\
\hline IV & Sulcus/gyrus & & $\begin{array}{c}0.93 \\
0.32 \mathrm{~mm}\end{array}$ \\
\hline $\mathbf{V}$ & $\begin{array}{l}\text { Irregular arrangement and size } \\
\text { of III, IIIs, IV type pit }\end{array}$ & & N/A \\
\hline
\end{tabular}

For the purpose of this paper, we will concentrate on the usage of the query format, and when we refer to ISO-15938-12:2008 (MPQF), we implicitly refer also to ISO-24800-3 (Part 3 of JPSearch). MPQF is an XML-based language in the sense that all MPQF instances (queries and responses) must be XML documents. Formally, MPQF is Part 12 of ISO/IEC 15938, "Information Technology - Multimedia Content Description Interface" better known as MPEG-7 (ISO/IEC 15938 Version 2, 2004). However, the query format was technically decoupled from MPEG-7 and is now metadata-neutral. One of the key features of MPQF is that expresses queries combining IR \& DR; being IR the expressive style of Information Retrieval systems (e.g. queryby-example and query-by-keywords) and DR the expressive style of XML Data Retrieval systems (e.g. XQuery (XQuery 1.0, 2006)), embracing a broad range of ways of expressing user information needs.

Regarding IR-like criteria, MPQF include but are not limited to QueryByDescription (query by example metadata description), QueryByFreeText, QueryByMedia (query by example media), Query$B y R O I$ (query by example region of interest), QueryByFeatureRange, QueryBySpatialRelationships, QueryByTemporalRelationships and QueryByRelevanceFeedback. Regarding DR-like criteria, MPQF offers its own XML query algebra for expressing conditions over the multimedia related XML metadata (e.g. Dublin Core, MPEG-7 or any other XML- based metadata format) but also offers the possibility to embed XQuery expressions.

The present paper is a positioning paper to demonstrate the feasibility of the Internet image search and discovery for diagnostic medical purpose. Results were based on a training-set of CEM-OB images annotated with specific CEM semantics and using the standardized multimedia query format for JPSearch ISO/IEC 24800

\section{MATERIAL AND METHODS}

Twenty five OB-CEM images obtained with a FICE (Fujinon Intelligent Chromoendoscopy) together with the resulting histological images were used in the training set. All were JPEG images annotated using standardized metadata for JPSearch ISO/IEC 24800 .

\subsection{IR System Metadata Description}

The information retrieval system Semantics of the Metadata was the classical modified Kudo criteria (Kiesslich et al., 2008) summarized in Table I. Annotation parameters include: Pit size, distance and regularity of normal round pits (typeI), detection of stellate or papillary images (type II), tubular/round pits smaller than type I (Type IIIs), Tubular large (type III), presence of sulcus /gyrus (type IV), irre- 
gular arrangement as size fo type III and IV (type V).

Whenever possible those parameters were automatically extracted by image analysis. See below.

\subsection{Image Search \& Retrieval Applica- tion}

Search and retrieval application built is an MPQF query processor. The software was limited to basic capabilities and did not provide yet CBIR functions

Query-by-Image formulation: According ISO15938-12:2008, the query-by-image is a combination of different condition expressions such as QueryByMedia, QueryByDescription, QueryByROI and SpatialQuery.

All these MPQF's condition types are based in the provision of an example (image, image region or image metadata description) expressing user information (see above IR system metadata). These condition types are selected or combined in order to return the best results.

\section{QueryByMedia}

Query-by-image (or simply query-by-example) similarly searches is a content based image retrieval (CBIR) technique (Lux et al., 2008) expressing user information with one or more example digital objects (e.g. an image file). Low-level features description instead of the example object bit stream is also considered query-by-example, in MPQF these two situations are differentiated, naming QueryByMedia to the first case (the digital media itself) and QueryByDescription the second one. In the first case is the query processor who decides which features to extract and use, and in the second case is the requester who perform the feature extraction and selection.

The MPQF's QueryByMedia type offers multiple possibilities to refer to the example media, as just including the media identifier (a locator such as an URL pointing to an external or internal resource) or directly embedding the image bit stream in Base64 encoding within the XML Query (see example in Code 1).

When the QueryByMedia type is used, it is up to the query processor to extract the proper low-level features to perform a similarity search over the index. MPQF does not specify which parameters or algorithms must be applied. In our case image analysis automatic extraction is done whenever possible

\section{QueryByDescription}

QueryByMedia and QueryByDescription are the fundamental operations of MPFQ and represent the query-by-example paradigm. The individual difference lies in the used sample data. The QueryByMedia query type uses a media sample such as image as a key for search, whereas QueryByDescription allows querying on the basis of an XML-based description.

For the purpose of the work described in this paper, we were using the QueryByDescription type to communicate to the server the specific metadata related to the example image fixed by the requester (e.g. pit size, distance and regularity of normal round pits, detection of stellate or papillary images, so on and so forth). These metadata were extracted whenever possible (by image analysis extraction) before submitting the query to the generic MPQF query processor.

\section{QueryByROI}

The MPQF's QueryByROI type extends the QueryByMedia type and describes a query operation that takes an example digital image as input and allows the specification of a region of interest. During the evaluation of this query type the region of interest is required to be considered for search. A region is defined by the IntegerMatrixType which allows the specification of a list of positive integer values describing individual points. The amount of necessary integer values per point is defined by the dim (dimension) attribute of the IntegerMatrixType type. If the dim attribute is set to two then two successive integer values specify one point in $2 \mathrm{D}$ space. The individual points define the region where for instance for $2 \mathrm{D}$, three points identify a triangle, four points a rectangular, and so on. The order of the individual points is contraclockwise. Code 2 gives an example of QueryByROI using a square bounding box.

For the purpose of the work described in this paper, we were using the QueryByROI type to offer to users the (optional) functionality to refine their query-by-image searches by specifying a region of interest (only a 2D square bounding box at the moment).

-The query processor only needed to crop the image according to the region specified and processed a conventional QueryByMedia evaluation. This way, the resulting images will be similar to the region specified.

-Furthermore, we considered to allow searching for "images containing region/s similar to the given one" and (if possible) to retrieve also the coordinates of these region/s. In despite of the fact that MPQF offers enough expressivity to formulate such a query, 


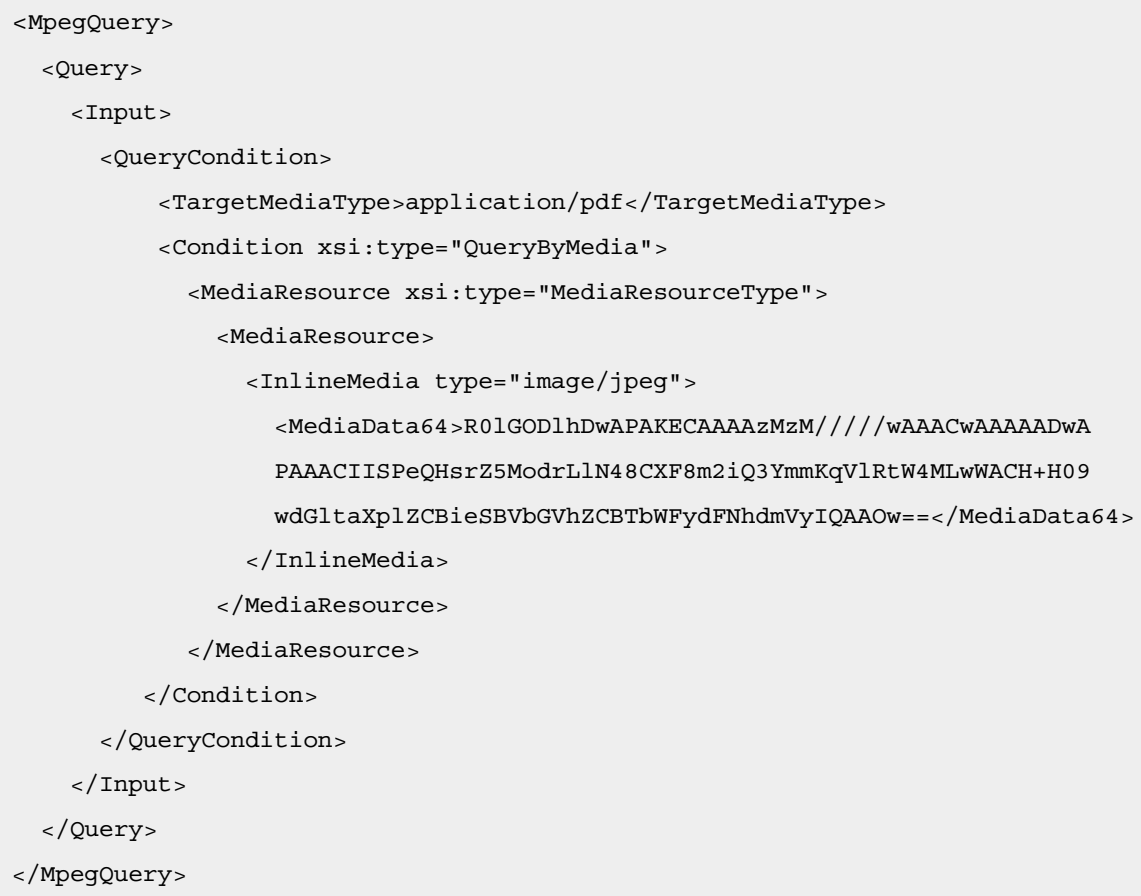

Code 1: QueryByMedia example.

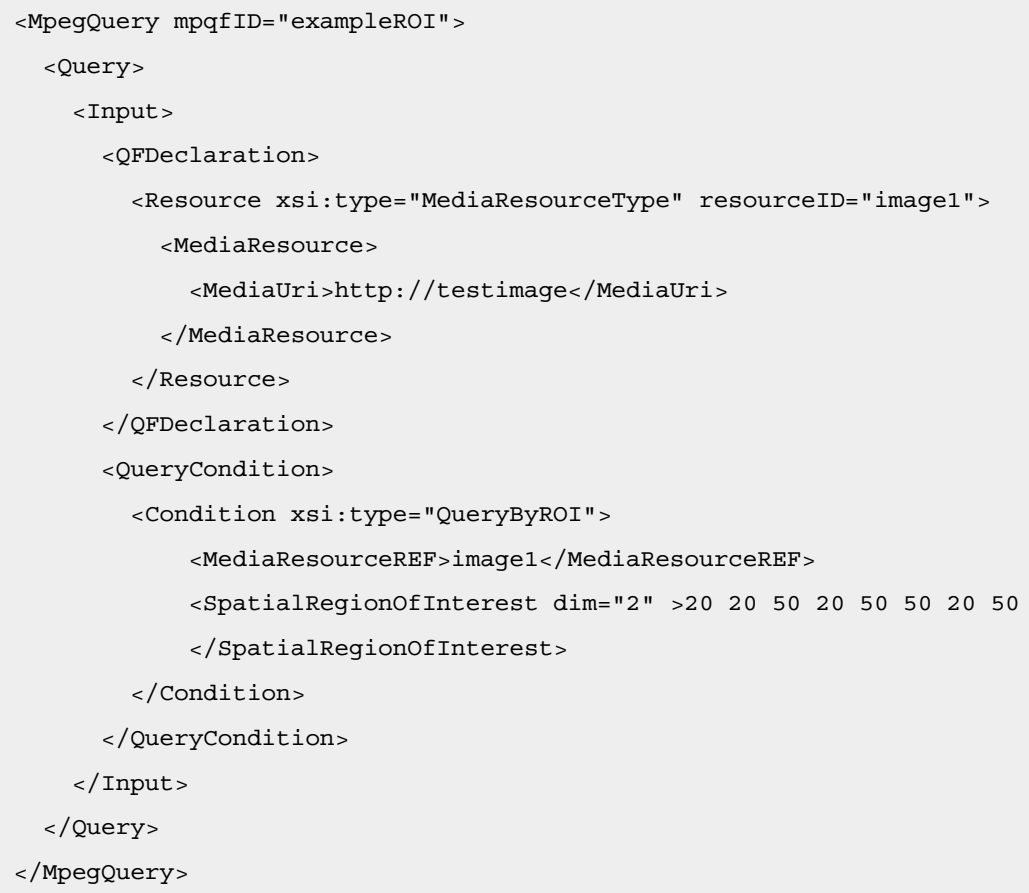

Code 2: QueryByROI example. 


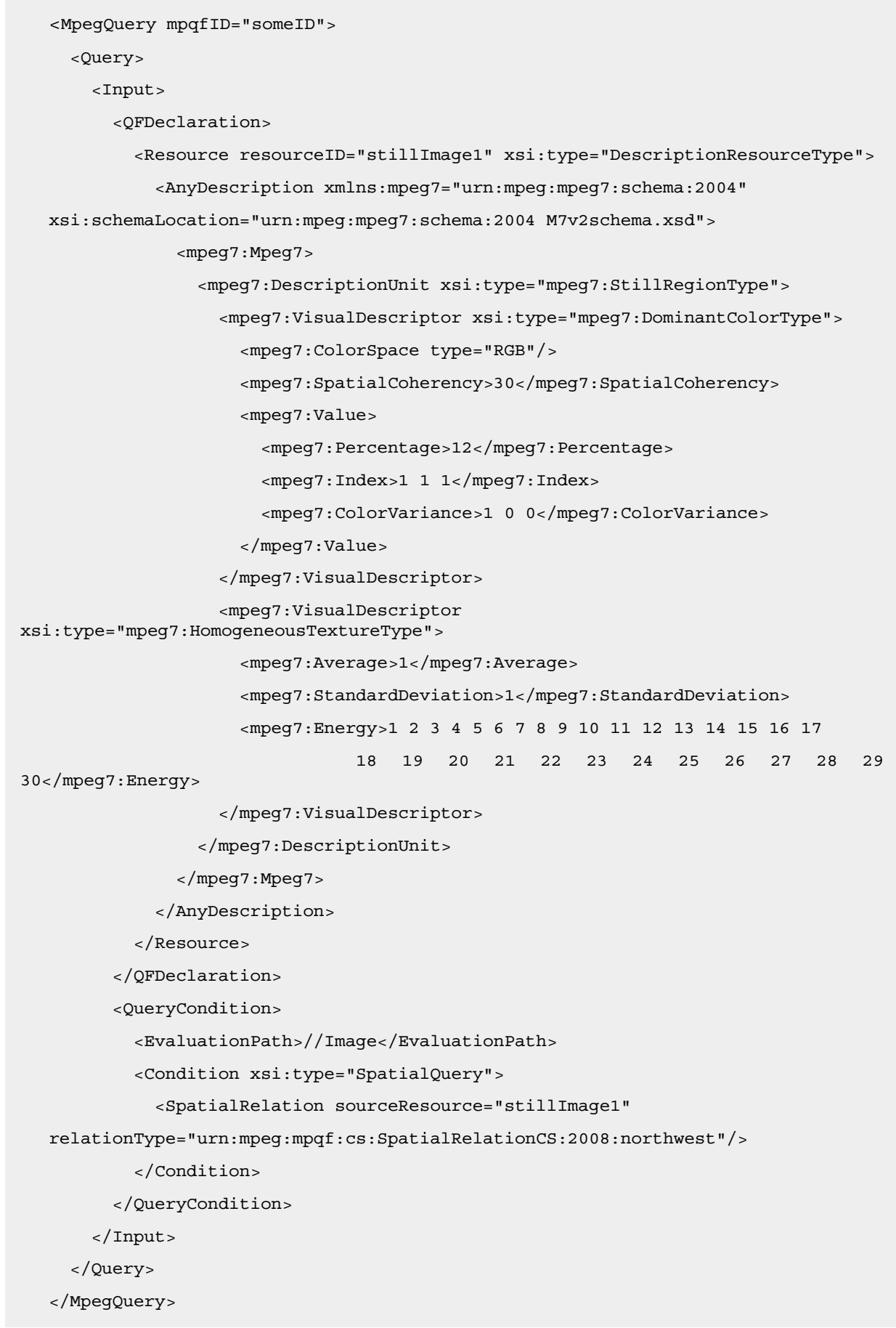

Code 3: SpatialQuery example.

unfortunately this interesting functionality is still subject to active research, and we cannot currently provide it

\section{SpatialQuery}

The MPQF's SpatialQuery type allows requests in the spatial domain where one or two regions (e.g., MPEG-7 StillRegion, etc.) are involved. Relationships among those regions and possible matching regions can be expressed by different relation types such as northOf, southOf, westOf, eastOf, contains, 
covers, overlaps, disjoint, so on and so forth. According to our knowledge, no CBIR query processors offer this kind of functionality; being the one we implemented an exception.

\section{RESULTS}

The provided user interface offers query-by-image also in combination with classic XML metadatabased criteria. Images are presented to a web application to be search in a local data-base, although the application aims the retrieval in Internet.

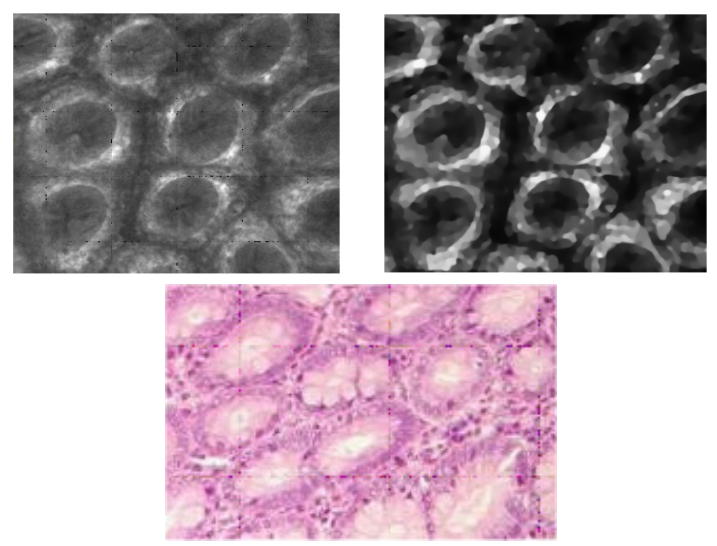

Figure 1: Automatic object identification and measurement. Find similar image in the data base including histological images. Normal colon.1- OB-CEM, 2- Image processing to extract parameters, 3- Histological image selection.
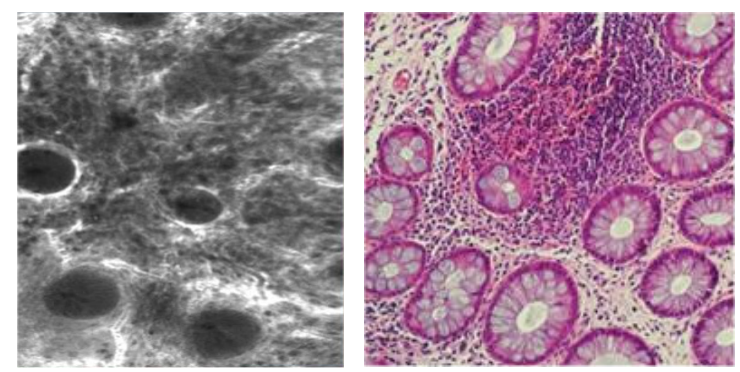

Figure 2: Colitis. Left. OB-CEM; Right- Histological image selection.

\section{DISCUSSION}

The present paper demonstrates that Internet search not only in Bibliographic but on image data-based could speed up medical diagnostic knowledge regarding novel technologies. This is the case of $\mathrm{OB}$ that is carried out by clinicians (endoscopists) while is based on microscopic morphology of the tissue, a domain specific of the surgical pathology.

To establish the gold standard in surgical pathology six are the main techniques 0: (1) Experience better then evidence; (2) Literature knowledge; (3) Scientific relevance or eminence (4) Interpretation (6) Personal impression. Therefore it is obvious that as soon as we collect sufficient experience and images available and accessed by pathologist, sooner the gold-standard for OB will be settle and incorporated into routine diagnostic procedures.

On this achievement the technique specify in the paper for image annotation and image query will be essential. It is, according to our knowledge, the first ISO-15938-12:2008 / ISO-24800-3 implementation. Although the authors (JD \& RT) had also contributed to the MPEG Query Format Reference Software \& Conformance (ISO/IEC 15938-12/Amd.1) with a basic MPQF processor, during the 88th MPEG meeting in Maui, USA, April 2009.

Many popular applications have now a day popularized the search for "similar images" (Google similar image; Gazopa; Zytel , etc.). Nevertheless, medical applications require more sophisticated techniques including specific medical semantics and domain ontology as explained in the present positioning paper. This is an unique and challenging field of applications for the ISO standards

\section{ACKNOWLEDGEMENTS}

This work has been partly supported by the Spanish government (TEC2008-06692-C02-01) and the CATAI association.

\section{REFERENCES}

Wang TD, VanDam J.(2004) Optical Biopsy: A New Frontier in Endoscopic Detection and Diagnosis. Clin Gastroenterol Hepatol 2(9): 744-753.

Ferrer-Roca O. (2008) Superresolution and Optical Biopsy. In CATAI 2009: Super-resolution and optical Biopsy. CATAI editions. Tenerife. Pp:45-54. ISBN: 978-84-612-8620-1.

Ferrer-Roca O. (2009) Endomicroscopia en anatomia patologica. Biopsia óptica. Rev.Esp.Patologia (accepted in 2009 and waiting to be published)

R. Tous (2008) Query formats for multimedia applications ISO/IEC 15938-12 (MPEG Query Format) \& ISO/IEC 24800 (JPSearch) In CATAI 2009: Super-resolution and optical Biopsy. CATAI editions. Tenerife. Pp2532 . 
Nawei Chen, Hagit Shatka, Dorothea Blostein, "Use of Figures in Literature Mining for Biomedical Digital Libraries," dial, pp.180-197, Second International Conference on Document Image Analysis for Libraries (DIAL'06), 2006

Natsu Ishii, Asako Koike, Yasunori Yamamoto, Toshihisa Takagi, "Figure Classification in Biomedical Literature towards Figure Mining," bibm, pp.263-269, 2008 IEEE InternationalConference on Bioinformatics and Biomedicine, 2008

Kiesslich R., Galle PR \& Neurath MF. Atlas of endomicroscopy. Springer-Verlag Heidelberg 2008. ISBN 978-3-540-34757-6

Hersch WR., Bhuptiraju RT., Ross L., Johnson P., Cohen AM., \& Kraemer DF. "TREC 2004 Genomics Track overview" Proc of TREC 2004 NIST Special Publication $2005 \mathrm{http} / / /$ ir.ohsu.edu/genomics

ISO/IEC 15938-12:2008 Information Technology - Multimedia Content Description Interface - Part 12:Query Format.

ISO/IEC 24800-3:2008 CD Information technology JPSearch - Part 3: JPSearch Query format. Output document from the 45th ISO/IEC JTC 1/SC 29/WG 1 Poitiers meeting, July 7th to 11 th, 2008

ISO/IEC 15938 Version 2. Information Technology Multimedia Content Description Interface (MPEG-7), 2004.

XQuery 1.0: An XML Query Language. W3C Proposed Recommendation 21 November 2006. See http://www.w3.org/TR/xquery/.

Mathias Lux, Savvas A. Chatzichristofis: Lire: lucene image retrieval: an extensible java CBIR library. ACM Multimedia 2008: 1085-1088 